\title{
A Case Report on Ipsilateral Autologous Limbal Transplantation \\ for Treatment of Corneal Stromal Scars
}

\section{Charuta J Puranik ${ }^{1,2 *}$, Lalitha Balla ${ }^{3}$, Falguni Pati ${ }^{4}$ and Shibu Chameettachal $^{4}$}

${ }^{1}$ Oculus Regenerus Eye Care and Research Center, Mehdipatnam Hyderabad, Telangana, India

${ }^{2}$ Virinchi-EYE, Ophthalmology Department, Virinchi Hospitals, Hyderabad, Telangana, India

${ }^{3}$ Sree Netralaya Eye Hospital and Lasik Centre, Hyderabad, Telangana, India

${ }^{4}$ Department of Biomedical Engineering, Indian Institute Technology Hyderabad

Kandi, Sangareddy, India

*Corresponding Author: Charuta J Puranik, Virinchi-EYE, Ophthalmology

Department, Virinchi Hospitals, Hyderabad, Telangana, India.
Received: April 11, 2020

Published: May 22, 2020

(C) All rights are reserved by Charuta J

Puranik.

\begin{abstract}
Introduction: Injury to cornea due to trauma or infection leads to stromal scarring and varying degrees of unaided and/or best corrected visual acuity loss posing a major challenge for the corneal specialist. Therapeutic options for superficial scars are rigid contact lenses or allogenic lamellar corneal transplantations which may fail due to graft rejection.

Aims: The primary aim of our case report is to demonstrate use of ipsilateral autologous limbal transplantation (IALT) in a case of mid stromal corneal scar resulting in effective resolution of the same.

Case Report: A 35-year-old male with a dense corneal scar, lagophthalmos and neurotrophy, with a healthy limbus, underwent ipsilateral autologous limbal transplantation. The scar gradually resolved and the clarity is maintained for past 40 months.

Discussion: Corneal stromal stem cells (CSSC) have been shown to reside in the human limbus and in the corneal stroma and have shown capacity to reverse corneal scaring in genetically engineered mice. Based on robust laboratory work from Funderburgh and others, we successfully transplanted autologous limbal chips in a human without manipulation effectually restoring corneal clarity and demonstrating over 40 months of stable outcomes.

Conclusion: We attribute the restoration to proliferation of resident CSSC induced by a conducive milieu provided from the limbal CSSC, or due to the migration of the CSSC from the limbal chips themselves. Functional keratocytes thus generated restored physiological collagen lamellae rendering the once scarred cornea transparent. The case also demonstrates an effective translation of years of meticulous bench side work to the clinic with a potential to benefit millions with corneal blindness.

Keywords: Ipsilateral Autologous Limbal Transplantation (IALT); Corneal Stromal Stem Cells (CSSC)
\end{abstract}

\section{Introduction}

The cornea is the clear transparent covering of the eye. Approximately $90 \%$ of the corneal volume is the stroma, a collagenous mesenchymal tissue composed of multiple lamellae of tightly packed parallel collagen fibrils. The small uniform diameter and orderly packing of stromal collagen is essential in transparency of the tissue. Injury to the cornea due to trauma or infection leads to irreversible stromal scarring and eventually varying degrees of unaided and/or best corrected visual loss. Corneal scars remain a major challenge for management for the corneal specialist [1].
Therapeutic option for management of superficial scars range from simple rigid contact lenses to complex component corneal surgery involving allogenic transplantation of corneal tissue from cadaveric donors and lifelong topical immunosuppression. Regardless of multiple advances medical and surgical, the commonest cause of corneal graft failure remains graft rejection [2].

Moreover, nebular or macular grade corneal scars which are not very dense or those accompanied by corneal neurotrophy are not amenable to traditional/component corneal transplant surgery. Others with transplants are frequently complicated by oc- 
currence of suture related complications including infections and more commonly astigmatism, thereby limiting the visual benefits to the patient. A significant proportion of these patients are young individuals in productive age group requiring binocular vision and sharp visual acuity. Rigid contact lenses do offer a significant option in not-so-dense scars with visual acuity 20/200 or better and in transplants with irregular astigmatism, however, many times patient acceptance for the same is limited. The lenses also worsen disability adjusted life years and a small proportion may not benefit from these at all [2]. Thus, most often faint scars remain untreated.

These limitations warrant search for alternative treatment modalities.

Cells responsible for scar deposition are fibroblastic cells derived from stromal keratocytes. Stem cells offer to regenerate tissue allowing functional restoration of tissue integrity. It has been shown that corneal stromal stem cells (CSSC) reside in the human limbus $[3,4]$. These CSSCs have demonstrated the capacity to reverse corneal scaring in animal models [5]. We propose that the CSSCs can be successfully transplanted in humans autologously thereby avoiding the side effects of an allograft, improving patient acceptance over contact lenses and giving lasting visual benefits.

\section{Hypothesis and scientific basis of procedure}

At the time of performing the clinical procedure described below, it was known that a small population of cells in the corneal stroma display mesenchymal stem cell like properties of clonal expansion, multipotency and display of stem cell specific markers. These Corneal Stromal stem cells (CCSC) had been identified and isolated and shown to reverse corneal opacity in mice by Dr James Funderburgh and his group in 2012. They also suggested that the limbus was a niche for these stem cells. Subsequently in 2014 his group showed that human limbal biopsy derived stem cells prevent scarring in mouse model [5].

The technique of simple limbal epithelial transplantation by $\mathrm{Dr}$ Sangwan ${ }^{6}$ where a strip of limbal palisade tissue is harvested and used autologously, has been in practice for treatment of epithelial limbal stem cell deficiency in chemical injuries, since year 2010. Visual loss in chemical injuries is due to the progressive conjunctivalization of corneal epithelium or due to non-healing large persistent epithelial defect. Some cases of chemical injury may be accompanied by corneal stromal scars, depending on the severity of initial injury. In their published outcomes of 125 cases, in addition to faster reepithelization of normal corneal epithelial phenotype this group reported progressive resolution of the stromal scars in the severe cases, as an serendipitous observation and attributed it to the likely presence of stromal stem cells in the limbal niche which had been inadvertently harvested along with the limbal epithelium. The technique attracted immense scientific scrutiny, invited many a critique and has managed to emerge as an effective, safe, reliable, and replicable surgical technique for treating ocular surface diseases due to limbal epithelial stem cell deficiency albeit in the absence of a clinical trial [6,7]. Encouraged by the experiments in mice and safety of process in humans, we decided to proceed with autologous ipsilateral simple limbal transplantation in the absence of ipsilateral stem cell deficiency, as a treatment modality for a case with superficial stromal scar.

\section{Case Report}

A 35-year-old male presented to us in May 2016 with right eye redness pain watering for 2 - 3 months. He has been diagnosed with a cerebellopontine angle tumour (Histopathological diagnosis of the excised specimen confirmed a Schwannoma) early in 2016 and had undergone craniotomy for excision of the same 2 3 months before presentation. At presentation, in the right eye he had visual acuity of counting fingers at $21 / 2 \mathrm{mt}$, lagophthalmos grade 5 (inability to close eyes despite maximum effort) exposure keratitis, absent corneal sensations and central corneal ulcer with a 2 - $3 \mathrm{~mm}$ oval epithelial defect and stromal infiltrate up to mid stroma with multiple deep stromal vessels extending into the ulcer from the limbus. Bells phenomenon was good. Left eye visual acuity was 20/20 N6, anterior segment and dilated fundus exams were within normal limits. Corneal scrapings of the right eye infiltrate were negative for any infective aetiology. He was diagnosed as a case of sterile corneal infiltrate secondary to exposure keratitis by the General ophthalmologist and lateral paramedian tarsorrhaphy was done immediately. The patient was started on topical therapy with tear substitute eye drops, topical chloramphenicol eye drops and 2\% HPMC gel. The ulcer gradually resolved in 2 months and on examination he had healed keratitis with a dense central corneal scar $\sim 3 \mathrm{~mm}$ x $4 \mathrm{~mm}$ size located centrally and overlying the pupil with deep vessels extending into the scar from 3 quadrants. Epithelium was stable (Fluorescein stain negative) and superior limbal palisades appeared healthy on slit lamp examination. Visual acuity at this point was 20/200 not improving with glasses or pin hole. Subsequently he was continued on tear substitutes for 4 months. The vision and scar density remained stable over these months and over these days he came to the clinic for visual rehabilitation requesting for a "new cornea". He was explained the risks of doing a transplant in a neurotrophic cornea and corneal transplant surgery was ruled out. Subsequently, in Dec 2016 simple limbal transplantation was done as follows. A 360-degree peritomy $2 \mathrm{~mm}$ from limbus was done including harvesting of ipsilateral limbal tissue 
from the superonasal limbus as a $2 \mathrm{~mm}$ strip. Epithelium over the entire cornea was debrided. Amniotic membrane was placed over the bare cornea and limbus and fixed with fibrin glue. The strip of limbal tissue was cut into 10 tiny bits which were placed evenly over the amniotic membrane on the scar area and droplets of fibrin glue were used to stabilize the bits. A bandage contact lens was applied over the eye. Post operatively the patient received topical antibiotics ( $\sim 4$ weeks) topical steroids (in tapering doses for 8 -10 weeks) and tear substitutes. Post-operative period was uneventful and visual acuity gradually improved to 20/120,20/60 and 20/30 and 1 month, 2 months and 3 months respectively and has been maintained since then, for the last 30 months or so. The scar density gradually reduced and the vascularisation reversed in 6 months with only faint ghost vessels visible at last follow up. The tarsorrhaphy was reversed at 24 months post simple limbal transplantation at which point he received an injection of Botulinum toxin from our oculoplastic surgeon following which there was no any change in the visual acuity or the scar despite grade 2 lagophthalmos.

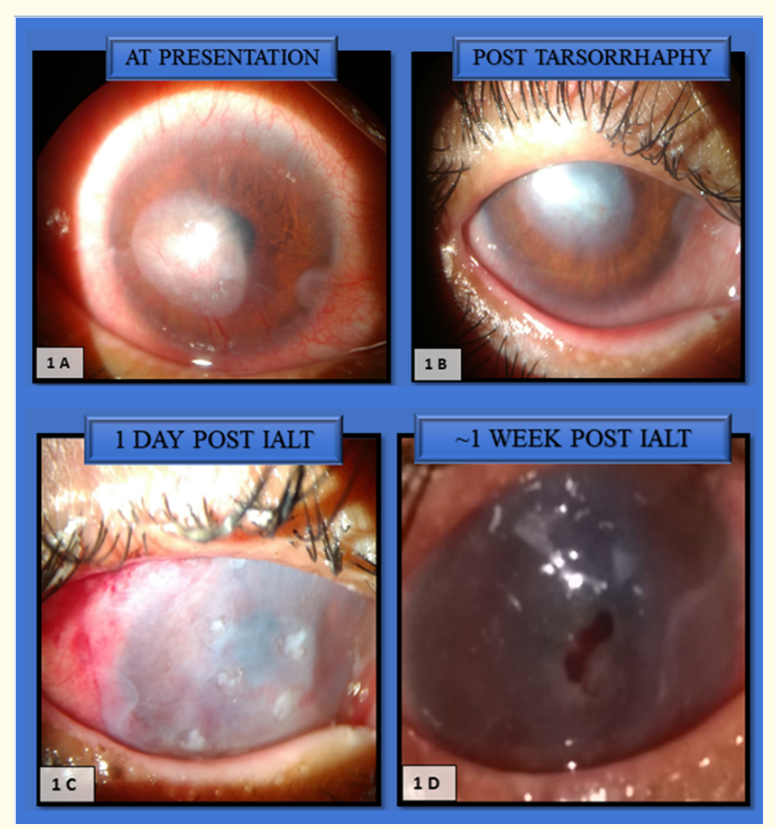

Figure 1: Serial Photographs from Preoperative to 1 week post Ipsilateral Autologous Limbal transplant (IALT). 1a: Dense central scar with 3 quadrant deep vessels obscuring visual axis after healing of neurotrophic ulcer $1 \mathrm{~b}$ : 5 months post lateral paramedian permanent tarsorrhaphy showing some reduction in calibre of deep vessels but scar density the same 1c: 1 day post IALT showing amniotic membrane and chips of Limbal tissue over scar area $1 \mathrm{~d}$ : 1 week post IALT showing gradual resorption of the amniotic membrane and the limbal chips.
To our knowledge this is the first-in-human cases of simple limbal stem cell transplant for a stromal scar in absence of limbal stem cell deficiency.

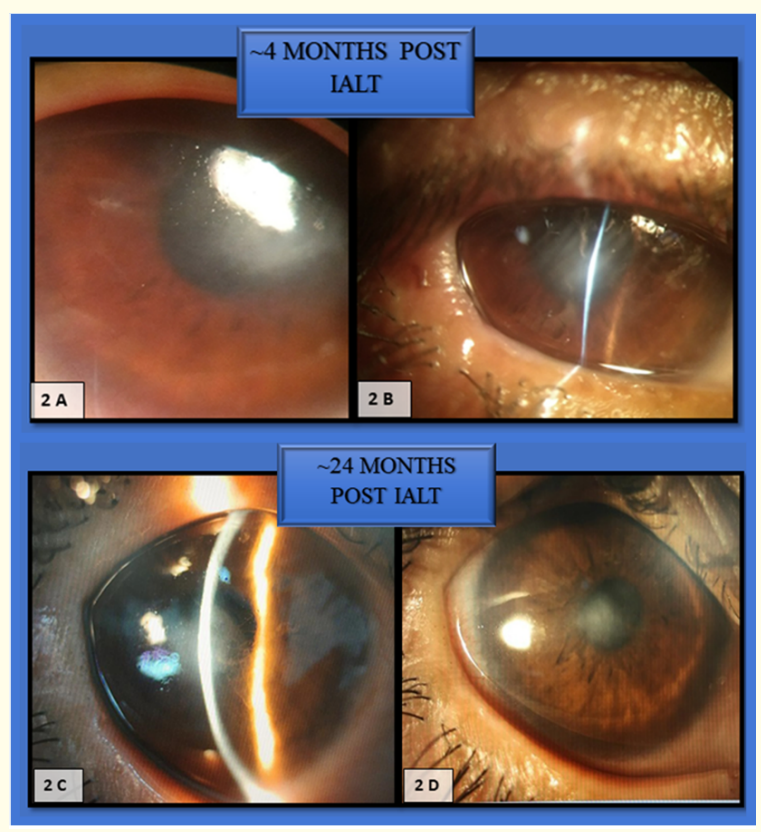

Figure 2: Serial Photographs post IALT. 2a and 2b: 4 months post IALT showing reduced scar density in diffuse and slit images. 2c and 2d: 24 months post IALT showing further reduction in scar density and appearance of ghost vessels.

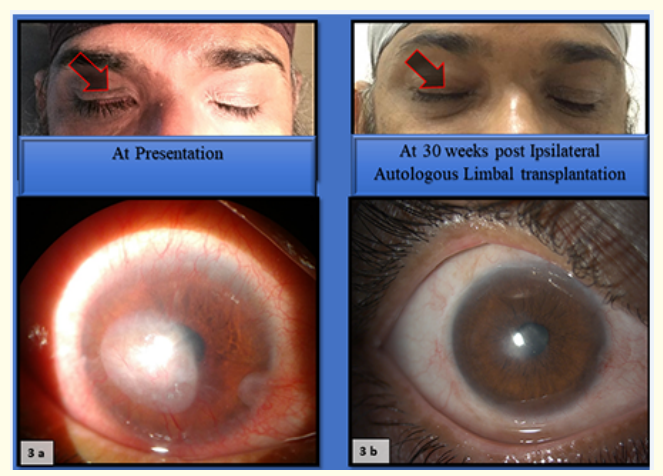

Figure 3: Pre and Post IALT photos. 3a: Masked Face photo demonstrating Lagophthalmos and diffuse slit lamp image showing initial dense vascularised scar. 3b: Masked face photo demonstrating no lagophthalmos at last follow up post tarsorrhaphy reversal and injection Botox and diffuse slit lamp image showing faint stromal scar at 30 months post IALT. 


\section{Discussion}

Migration of corneal stromal stem cells vs keratocytes

Corneal stroma is a collagenous mesenchymal tissue with tightly packed fibrils. The keratocytes are a population of stromal cells, sandwiched between the lamellae, responsible for secretion of the stromal extracellular matrix composed of tissue specific keratan sulphate proteoglycan (KSPG). KSPG along with the collagen, together render the cornea transparent [8-12]. Stromal keratocytes are neutral crest derived mesenchymal cells which are in the quiescent phase of the cell cycle [6] and stay as such in their life except in response to trauma or infections. Such events trigger a fibroblastic transformation and transformed cells then express $\alpha$ smooth muscle actin and an array fibrotic extracellular matrix $[13,14]$ which render the cornea non transparent which may remain for decades. In-vitro studies have shown that keratocytes reach replicative senescence after 70 to 80 population doublings when derived from clonal populations of adult bovine stromal cells and subjected to reduced mitogen culture media [14]. These findings add weight to evidence that some keratocytes maintain regenerative potential. These cells, now called the CSSC Corneal stromal stem cells (CD73, CD90 and PAX6 positive) can replicate in vitro to keratocytes without undergoing fibroblast transformation for many generations [3] and have shown the capacity to restore corneal transparency in mouse corneal scar models [15].

Corneal epithelial cells undergo far more wear and tear than stromal cells and a constant source for replenishment has been shown to be present in the corneal limbus in the form of corneal epithelial stem cells present in the limbal niche at the palisades of Vogt [16].

These CESC have been harvested form the limbus by surgical dissection and on cell cultures shown to be accompanied by a small number of mesenchymal cells demonstrating markers similar to CSSCs (CD105, CD106, CD54, CD166, CD90, CD29, and CD71 and PAX6 positive) [17] and to mesenchymal stem cells from bone marrow [18-21].

Limbal CSSC modulate the microenvironment when co cultured with CESC that show better expansion in their presence [20]. In vivo, Iyer., et al. demonstrated outcome of allogenic simple limbal epithelial transplantation (alloSLET) in eyes in the early stage following ocular chemical injury to achieve rapid epithelialisation. In their series, none of the eyes developed stromal scarring warranting penetrating keratoplasty [22].
Aim of regenerative medicine has been to resurrect normal anatomy and physiology of the dysfunctional tissue at a cellular subcellular and genetic level [23]. It comprises of utilising diverse therapeutic modalities to achieve this goal. Use of bioengineered cultured cells also called the "living drugs" is a complex technology $[24,25]$. Despite the complexities, cell-based technologies using tissue engineering have offered new therapies for many conditions necessitating isolation of the cells their expansion in vitro maintaining their cellular phenotypic and functional characteristics. However, recently Dr Atala's group has successfully shown that tissue morphogenesis can be controlled in vivo by delivering crucial signals to either the infiltrating multipotent cells or the resident tissue specific progenitor cells leading to formation of functional tissues in situ as against the conventional cell-based strategies [2629].

In an interesting paper from Lee., et al. [26] the authors demonstrated recruitment of host renal stem/progenitor cells within an injured kidney (ischemia/reperfusion injury) in an animal model, that facilitated anatomical and physiological recovery. The authors elegantly demonstrated the regenerative effect and attributed the same to a combination of host response to the injury and the collagen hydrogel that was injected into the animal kidney, that eventually led to a promotion of repair reduced cell death and decreased post injury glomeruli loss. They also attributed mobilisation of somatic stem cells to injured tissues that differentiate into tissue specific lineages and participating in the repair process as has been demonstrated in other studies [30-32].

\section{Conclusion and Future Directions}

We propose that through the process of Simple limbal transplantation, resident CSSC in the corneal stroma that are capable of expansion and self-renewal were likely induced due to a conducive milieu provided from the limbal CSSC, amniotic membrane and the fibrin. This in turn generated functional keratocytes producing physiological collagen lamellae rendering the once scarred cornea transparent. We have started the process of a clinical trial to demonstrate efficacy of simple limbal stem cell transplantation over non-intervention (REF/2019/03/024811). Further studies would also be needed to understand the homing of limbal CSSC and if other sources of mesenchymal stem cells (such as umbilical cord derived stem cells or BM derived stem cells) can be used for treatment of corneal scars in vivo. Also unanswered is the role of the blood vessels that regressed with the resolution of the scar in this case and if the same had contributed somatic MSC to the repair process. 


\section{Author Contributions}

Charuta J Puranik: Conception and design, Financial support, Collection and assembly of data, Data analysis and interpretation, Manuscript writing, Final approval of manuscript.

Lalitha Balla: Administrative support, Provision of study material or patients, Manuscript writing.

Falguni Pati, Shibu Chameettachal: Data analysis and interpretation, Manuscript writing.

\section{Funding}

The study was supported by a grant from Lopamudra Charitable trust.

\section{Conflicts of Interest}

The authors declare that they have no conflicts of interest.

\section{Ethical Approval}

This article does not contain any studies involving animals performed by any of the authors.

All procedures performed in human participants were in accordance with the ethical standards of the national research committee and with the 1964 Helsinki Declaration and its later amendments. The authors retrospectively sought ethics approval and the same was waived off as the procedure has been safely performed for patients with limbal stem cell deficiency.

Informed consent was obtained from all individual participants involved in the study.

\section{Data Availability Statement}

The authors confirm that the data supporting the findings of this study are available within the article and its supplementary materials.

\section{Bibliography}

1. Mariotti SP. "Global Data on Visual Impairments". World Health Organization (2010).

2. ThompsonRW Jr., et al. "Long-term graft survival after penetrating keratoplasty". Ophthalmology 110.7 (2003): 13961402 .

3. Du Y., et al. "Multipotent stem cells in human corneal stroma". Stem Cells 23 (2005): 1266-1275.

4. Polisetty N., et al. "Mesenchymal cells from limbal stroma of human eye". Molecular Vision 14 (2008): 431-442.
5. Basu S., et al. "Human limbal biopsy-derived stromal stem cells prevent corneal scarring". Science Translational Medicine 266 (2014): 266ra17.

6. Basu S., et al. "Simple limbal epithelial transplantation: longterm clinical outcomes in 125 cases of unilateral chronic ocular surface burns". Ophthalmology 123 (2016): 1000-1010.

7. Search criteria "simple+limbal+epithelial+transplantation+li mbal+stem+cell+deficiency".

8. Parfitt GJ., et al. "Three-dimensional reconstruction of collagen-proteoglycan interactions in the mouse corneal stroma by electron tomography". Journal of Structural Biology 170 (2010): 392-397.

9. Lewis PN., et al. "Structural interactions between collagen and proteoglycans are elucidated by three-dimensional electron tomography of bovine cornea". Structure 18 (2010): 239-245.

10. Hassell JR and Birk DE. "The molecular basis of corneal transparency". Experimental Eye Research 91 (2010): 326-335.

11. Kao WW and Liu CY. "Roles of lumican and keratocan on corneal transparency". Glycoconjugate Journal 19 (2002): 275285.

12. Funderburgh JL. "Keratan sulfate: Structure, biosynthesis, and function". Glycobiology 10 (2000): 951-958.

13. Funderburgh JL., et al. "Keratocyte phenotype mediates proteoglycan structure: A role for fibroblasts in corneal fibrosis". Journal of Biological Chemistry 278 (2003): 45629-45637.

14. Funderburgh ML., et al. "PAX6 expression identifies progenitor cells for corneal keratocytes". The FASEB Journal 19 (2005): 1371-1373.

15. Du Y., et al. "Stem cell therapy restores transparency to defective murine corneas”. Stem Cells 27 (2009): 1635-1642.

16. Chen JJ and Tseng SC. "Corneal epithelial wound healing in partial limbal deficiency". Investigative Ophthalmology and Visual Science 31 (1990): 1301-1314.

17. Polisetty N., et al. "Mesenchymal cells from limbal stroma of human eye". Molecular Vision 14 (2008): 431-442.

18. MJ Branch., et al. "Hopkinson, Mesenchymal stem cells in the human corneal limbal stroma". Investigative Ophthalmology and Visual Science 53 (2012): 5109-5116. 
19. GG Li., et al. "Mesenchymal stem cells derived from human limbal niche cells". Investigative Ophthalmology and Visual Science 53 (2012): 5686-5697.

20. N Polisetty., et al. "Mesenchymal cells from limbal stroma of human eye". Molecular Vision 14 (2008): 431-442.

21. LJ Bray., et al. "Immunosuppressive properties of mesenchymal stromal cell cultures derived from the limbus of human and rabbit corneas". Cytotherapy 16 (2014): 64-73.

22. Iyer G., et al. "Outcome of allo simple limbal epithelial transplantation (alloLSET)in the early stage of ocular chemical injury". British Journal of Ophthalmology 101 (2017): 828-833.

23. Trommelmans L. "The challenge of regenerative medicine". Hastings Center Report 40.26 (2010): 24.

24. Daar AS and Greenwood HL. "A proposed definition of regenerative medicine". Journal of Tissue Engineering and Regenerative Medicine 1 (2007): 179-184.

25. Committee for Advanced Therapies (CAT), CAT Scientific Secretariat Schneider CK., et al. "Challenges with advanced therapy medicinal products and how to meet them". Nature Reviews Drug Discovery 9 (2010): 195-201.

26. Lee SJ., et al. "Host cell mobilization for in situ tissue regeneration”. Rejuvenation Research 11 (2008): 747-756.

27. Ju YM., et al. "In situ regeneration of skeletal muscle tissue through host cell recruitment". Acta Biomaterialia 10 (2014): 4332-4339.

28. Ko IK., et al. "Combined systemic and local delivery of stem cell inducing/recruiting factors for in situ tissue regeneration". The FASEB Journal 26 (2012): 158-168.

29. Ko IK., et al. "In situ tissue regeneration through host stem cell recruitment". Experimental and Molecular Medicine 45 (2013): e57.

30. Asahara T., et al. "Isolation of putative progenitor endothelial cells for angiogenesis". Science 275 (1997): 964-967.

31. Crosby JR., et al. "Endothelial cells of hematopoietic origin make a significant contribution to adult blood vessel formation". Circulation Research 87 (2000): 728-730.

32. McKay R. "Stem cells-hype and hope". Nature 406 (2000): 361364.

\section{Assets from publication with us}

- Prompt Acknowledgement after receiving the article

- Thorough Double blinded peer review

- Rapid Publication

- Issue of Publication Certificate

- High visibility of your Published work

Website: www.actascientific.com/

Submit Article: www.actascientific.com/submission.php Email us: editor@actascientific.com

Contact us: +919182824667 